\title{
and anticipated impacts.
}

\section{T Charles Witzel ${ }^{1}$, Fiona M Burns ${ }^{2}$, Peter Weatherburn ${ }^{1}$, Alison J Rodger ${ }^{2}$.}

1 Sigma Research, London School of Hygiene \& Tropical Medicine. 2 Research Department of Infection \& Population Health, University College London.

\section{Introduction}

HIV self-testing (HIVST) is a new approach, enabling individuals to test themselves for HIV in a location and at a time of their choosing using a rapid diagnostic test (1-3). The first HIVST came to market in the UK in 2015 and since then there have been a variety of implementation projects (4-6).

HIVST has the potential to increase uptake and frequency of HIV testing for those most at risk (3). Most recently, SELPHI, an online randomised controlled trial (RCT) delivering an HIVST intervention, has begun enrolling men who have sex with men (MSM) and transgender people in England and Wales.

Study aims: To understand the perspectives of key informants on the implementation of HIVST.

\section{Methods}

In order to inform development of an HIVST intervention for use in the SELPHI RCT, 17 in-depth interviews were conducted with key informants (KIs) including clinical staff in NHS HIV and sexually transmitted infection (STI) services, voluntary sector service providers and HIV testing commissioners from across England. Table 1 describes our sample.

\section{- Data collected between April and December 2016}

- Interviews were face-to-face and over the telephone.

-Audio recorded and transcribed verbatim.

- Thematic framework analysis (7).

\section{$\underline{\text { Results }}$}

\section{Increasing patient choice}

HIVST was valued for increasing choice in accessing HIV testing. Specifically, those who directly provided HIV testing services acknowledged that the remote nature of the technology might increase accessibility for some, potentially expanding testing to new audiences currently experiencing structural barriers.

Local context \& client group

Careful attention to intervention design was identified as important, as local context and client group shaped anticipated patient responses to HIVST. Several KIs expressed concern that a national HIVST intervention could be of limited utility if developed with insufficient attention to local needs. When considering intervention components, a KI involved in community outreach programmes in public sex environments stated:

Whether it's easy to say groups or whether it's about circumstances, because there are going to be some people who can't have a test delivered to their home, or even take a test home. [...] If you look at the MSM community [in the South West of England], the large majority of MSMs have a partner or a wife or children, and lead a heterosexual lifestyle, so may not want to even think about bringing any kind of testing kit into their home. (Service delivery voluntary sector. South West England).

\section{References} I think the big benefit to self-
testing is the ability for people to testing is the ability for people to that they choose to, so a choice is reach people in more rural communities and places where delivery, voluntary sector. North of England).

\begin{tabular}{|c|c|c|}
\hline Sector & Role & Numbers \\
\hline \multirow[t]{2}{*}{ Voluntary } & Service delivery & 3 \\
\hline & Strategic & 4 \\
\hline \multirow[t]{2}{*}{ Commissioning } & Local & 1 \\
\hline & National & 1 \\
\hline \multirow[t]{3}{*}{ Clinical } & Clinician & 4 \\
\hline & Health advisor & 2 \\
\hline & Nurse & 2 \\
\hline
\end{tabular}

Integration \& pathways to care

It was widely perceived that HIVST should be delivered through integrated approaches that provide direct pathways into additional services and HIV care. While some felt HIVST should be delivered through clinics alongside other STI tests, others felt that the most important element was the development of multiple pathways which responded to patient need. Flexibility was highly valued.
I think if you can set up a variety of potential openings and opportunities and routes into engagement, whether that's community clinics or different healthcare settings and services, then people will gravitate to the one that's most suitable for them, either logistically or geographically or psychologically. So I think it's multi-pronged, really. (Clinician, North West of England)
Anticipated impacts

A range of impacts were predicted by KIs. The primary concerns centred on dislocation of HIV testing from supportive structures thereby leading to an increase in bacterial STIs through reduced opportunities for prevention and STI detection. Clinical staff specifically were concerned about a potential for increased selfharm. Some felt these impacts would occur in a very small group, while others believed they would be widespread consequences of service delivery. Conversely, this dislocation offered the potential for empowerment for some patients and was valued for increasing autonomy, and potentially increasing accessibility.

I think the benefits [of HIVST] are that it increases that nation's selfcare and agency. For me, people have the right to know what's going on for them. They should be able to do that in any way that technology allows. Obviously, there are people who just want to have tests and don't want to tell anybody. (Strategic role, voluntary sector, North West England).

\section{Discussion}

In this qualitative in-depth study with $17 \mathrm{Kls}$, a number of key themes emerged providing conditional support for HIVST implementation. HIVST interventions should be responsive to context, taking into account both local and national needs. The flexibility of HIVST interventions to provide a variety of entry points into further STI testing and into HIV care may be a key benefit of a wider-roll out. Innovative approaches in this regard should be investigated. Concerns centred on negative impacts of dislocating HIV testing from supportive structures. Our results indicate that innovative service delivery designs which address these concerns while harnessing the benefits of flexibility may be key to KI buy-in for HIVST implementation and improved patient outcomes. 